\title{
Leakage of Hepaticojejunal Anastomosis: Radiological Interventional Therapy
}

\author{
Katharina May Peter Hunold \\ Clinic for Radiology and Nuclear Medicine, University Hospital Schleswig-Holstein, Campus Lübeck, Lübeck, Germany
}

\section{Keywords}

Intervention - Drainage implant .

Percutaneous transhepatic biliary drainage, PTBD .

Hepaticojejunostomy

\section{Summary}

Background: Hepaticojejunostomy is an established procedure accompanying liver resection as well as hepatobiliary and pancreatic surgery. Typical complications requiring radiological intervention are abscesses and anastomosis leakage. Biliary obstruction and strictures are less frequent indications for interventional radiology since many of them can be treated endoscopically. Methods: Depending on anatomic location, underlying etiology, and complication of leakage, different procedures of interventional radiology are performed: treating abscesses through percutaneous abscess drainage (PAD), handling biliary leakage by percutaneous transhepatic biliary drainage (PTBD) after percutaneous transhepatic cholangiography (PTC), or rarely performed percutaneous stent implantation and internalization after PTC and PAD to treat biliary strictures or to cover the leak. A selective literature search was performed, taking into account recent papers of radiological interventions concerning leakage of hepaticojejunostomy. Results: Different radiological interventions for the treatment of potentially devastating complications after hepaticojejunostomy are recommended. PAD and PTBD serve either as a definite treatment or as bridging therapy until re-surgery is performed. Complications mainly concern uncontrollable bleeding during the intervention. Conclusion: Radiological interventions are safe and usable if the indication is meticulously chosen. PAD is one of the most commonly performed procedures by interventional radiologists and a well-established and safe procedure. PTC,
PTBD, and biliary stent implantation are important procedures which are required if endoscopic interventions failed or cannot be performed due to postoperatively changed anatomical structures.

(c) 2017 S. Karger GmbH, Freiburg

\section{Introduction}

Hepaticojejunostomy is an important component of hepatic, hepatobiliary and pancreatic surgery. The first report of a choledochoenterostomy was published in 1891 [1]. Back then, deaths occurred related to bile leaks [1]. By now, the knowledge, techniques, and circumstances (e.g. antibiotics) have improved, and major surgery is more common and less risky now [2].

However, leakage of anastomosis, secondary infection, or bleeding following major surgery are still possible today and require rapid treatment. Complete healing takes time, particularly that of anastomosis leakage and biliary strictures, and therefore longerlasting treatment methods are needed to avoid recurring surgery. According to the guidelines of the Infectious Diseases Society of America (IDSA) regarding complicated intra-abdominal infections of adults and children from 2010, intra-abdominal infection is the second most common cause of all infectious mortality in intensive care units in the USA [3]. Current indications for hepaticojejunostomy are injuries to the biliary system, benign or iatrogenic strictures, and obstruction caused by pancreatic or duct wall tumors [1]. Rare indications are trauma or dilated areas in sclerosing cholangitis [1].

Since the advent of image-guided percutaneous abscess drainage (PAD) as early as the mid-1970s [4], there was a constant development of technical advices, percutaneous techniques, and available materials, leading to a higher frequency and a better outcome in interventional radiology. Together with broad-spectrum

\section{KARGER}

() 2017 S. Karger GmbH, Freiburg

Fax +497614520714 
antibiotics they led to more treatment opportunities. Procedures are established by now, and many abscesses that were previously considered to be best treated by laparotomy are now within reach of interventional radiology [4-7]. Whenever accessible, percutaneous drainage of abdominal abscesses and well located fluid collections should be preferred to surgical drainage [4].

PAD is currently an accepted treatment for many intra-abdominal infections $[4,6,8,9]$. Improvements were made in the use of broad antibiotics and surgical skills, resulting in a wider spectrum of patients undergoing complex surgical operations such as hepaticojejunostomy. Therefore, radiological interventions of postoperative complications are even more important than ever. They reduce morbidity and mortality compared with open surgical drainage by giving the opportunity of minimally invasive drainage after noninvasive detection of bile leaks [7, 8]. Furthermore, avoidance of general anesthesia in most cases proves beneficial for many seriously ill patients [7-10]. Radiological interventions provide the opportunity of optimizing the patient's condition before repeated operation or function as a definitive treatment $[7,9]$. High availability of diagnostic imaging simplifies the planning and performance of minimally invasive therapies. Depending on the location, technical success rates for PAD of up to $93 \%$ are described in the literature [5-10].

\section{General Preparation}

General preparation prior to radiological interventions after hepaticojejunostomy is the same whether performing PAD in computed tomography (CT) or percutaneous biliary drainage and stent implantation in angiography.

\section{Primary Imaging}

In order to define the indication, primary imaging using sonography, CT, or magnetic resonance imaging should be performed, covering the whole expansion of the abscess and its exact anatomical position relationships [4-9]. Furthermore, it is important to realize complicating elements such as fistulas, septations, and the number of abscesses with possible communications to each other. To plan the perfect access route, it should be ensured that also the area surrounding the abscess is fully depicted and that there is adequate distance to vessels, critically parenchymal organs, and interposed structures such as bowel $[5,7]$.

$\mathrm{CT}$ is the method of choice when diagnostic imaging is urgently needed. Due to low interobserver variability, high availability, and fast acquisition time, CT is advantageous for guiding drainage, particularly for small and deep abscesses in close spatial relationship to vital structures and presentation of complex abscesses $[8,11]$. Intravenous and oral contrast simplify the differentiation of the abscess and its surroundings $[5,7]$. Measuring the Hounsfield units (HU) within the abscess provides helpful information on the characterization of the fluid in order to choose the right lumen size of the drain. High viscosity of fluid reduces the chances of success [8]. Even though a reliable differentiation between abscess, biloma, and sterile fluid is not always possible by CT, sonography may help in differentiation since sterile fluid collections are anechoic [8]. Close position to important structures, i.e. bowel and huge vessels, also complicate the intervention. Transpleural puncturing of subphrenic fluid collections and abscesses have a higher risk for pleural empyema through dislocation of germs during the intervention $[5,8]$.

The indication for radiological intervention should be discussed in interdisciplinary consent between surgeon, endoscopically working internist, and radiologist and depend on the abilities [5, 8]. Planning of the intervention can either be made on the basis of primary imaging if performed directly prior to the intervention, usually mapping the whole abdomen, or alternatively as a selective scan if the primary imaging has been performed earlier.

\section{Clinical Situation and Patient's History}

Previous history of allergic reactions to contrast media and local anesthetics as well as function of kidneys and thyroid gland should be queried prior to the intervention. In individual cases, however, clinical symptoms pointing to inflammation or prolonged bleeding should be inquired about [5].

Before any intervention, current laboratory parameters such as the international normalized ratio (INR), prothrombin time, thrombocyte count, leukocytes, creatinine, and thyroid-stimulating hormone should be available. In case of any manifest difficulty concerning the blood coagulation status (INR $>1.5$, thrombocytes $<50,000 / \mu$ l, prothrombin time $>20 \mathrm{~s}$ ), appropriate countermeasures have to be discussed. Antiplatelet medications (i.e. ASA, clopidogrel, and Coumadin) should be stopped. Liver and renal function parameters should be known, since contrast material and other drugs (i.e. antibiotics) may influence liver and renal function. Vital parameters (blood pressure, oxygen saturation, pulse) need to be monitored during the intervention $[5,6]$.

\section{Contraindications}

If there is no safe access route to the abscess as well as a high risk of surrounding injury, such as bowel perforation, one should refrain from intervention [5]. Nevertheless, there is no absolute contraindication for PAD and percutaneous transhepatic biliary drainage (PTBD). Relative contraindications are lack of proper blood coagulation, sterile collections, or rejection by the patient [5, $6,8,10,12]$. Puncturing sterile fluid collections can lead to a secondary infection through prolonged catheter drainage. Access routes that require a lesion of the pleura bear the risk of pneumothorax, pleural effusion, or pleura empyema and should therefore be avoided whenever possible $[8,10]$.

\section{Sedation}

In most cases local anesthesia is adequate for intervention. Conscious sedation may decrease the risk of procedure-related complications by reducing pain and anxiety of the patient and by comforting the treating radiologist $[5,6,8]$. Young children and uncooperative patients benefit from general anesthesia [9]. 


\section{Selected Radiological Interventions}

Computed Tomography-Guided Percutaneous Abscess Drainage

$\mathrm{PAD}$ is one of the most commonly performed procedures in interventional radiology. For the treatment of sepsis and the patient's relief of symptoms, puncture is used to distinguish between purulent fluid, blood, bile, lymph, or pancreatic secretions [5, 9]. Furthermore, obliteration of recurring cysts or collections of sclerosing agents is possible [9]. The optimal pathway for PAD is a short direct route to the target object that does not touch vital structures. An extraperitoneal approach to reduce the risk of peritoneal contamination is preferred [9]. In general, there are three different techniques for PAD: direct puncture, tandem trocar technique, and Seldinger technique $[6,8]$. In the case of perihepatic/peripancreatic location of abscesses as a complication of hepaticojejunostomy, PAD are mostly performed in Seldinger technique since fluid collections tend to be distant to the puncture site or come along with limited access. Direct puncture and tandem trocar technique should only be exceptionally performed in the epigastrium for large superficial fluid collections with a safe and short access route and are not further explained. The drainage system may consist of a pigtail or Malecot basket drainage. The advantage of the pigtail catheter is a coiled head that will hold the drain in position, and its many holes at the distal end makes fluid run off easily with a smaller chance of clotting within the drain. The advantage of the Malecot basket drainage is its straight shape that can be easily placed in small thin fluid collections.

Technical success rates of drainage of peripancreatic abscesses are as high as $70-100 \%$ [8]. Major complications described include hemorrhage, septic shock, enteric fistula, peritonitis, and hemopneumothorax. Severe bleeding after puncture of blood vessels may require embolization, surgery, or blood transfusion. Minor complications include pain, minor bleeding, infection, and pericatheter leakage $[5,6,8]$. The overall complication rate reported is less than $15 \%$ [9]. It can be expected that minor complications predominate.

Daily catheter inspection is an important follow-up after PAD to detect pericatheter leakage, local skin complications, and other complications related to the intervention. Orders should be written to regularly flush the catheter with saline every $8 \mathrm{~h}[6]$. The catheter is usually removed when drainage suspends at less than $20 \mathrm{ml} /$ day. Repeat imaging is only needed if the patient's condition does not improve [6]. Early removal of the catheter will increase the recurrence rate of abscess between 8 and 20\% [6]. Other reasons for recurring abscess are undetected fistula or drainage of tumor abscess [6].

\section{Percutaneous Transhepatic Cholangiography and Internal-}

External Percutaneous Biliary Drainage

Biliary leakage is a common complication after liver and biliary surgery such as hepaticojejunostomy [13]. With the development of laparoscopic techniques and liver transplantation the rate of biliary leakage is reported to range from 0.8 to $12 \%$ [13]. The leaks mainly occur at the biliary-enteric anastomotic site $[1,13]$ and may result in biliary peritonitis with subsequent abscesses, bilomas, or fistulas [13]. Bilomas should be drained with either perioperatively placed drainage or PAD [13].

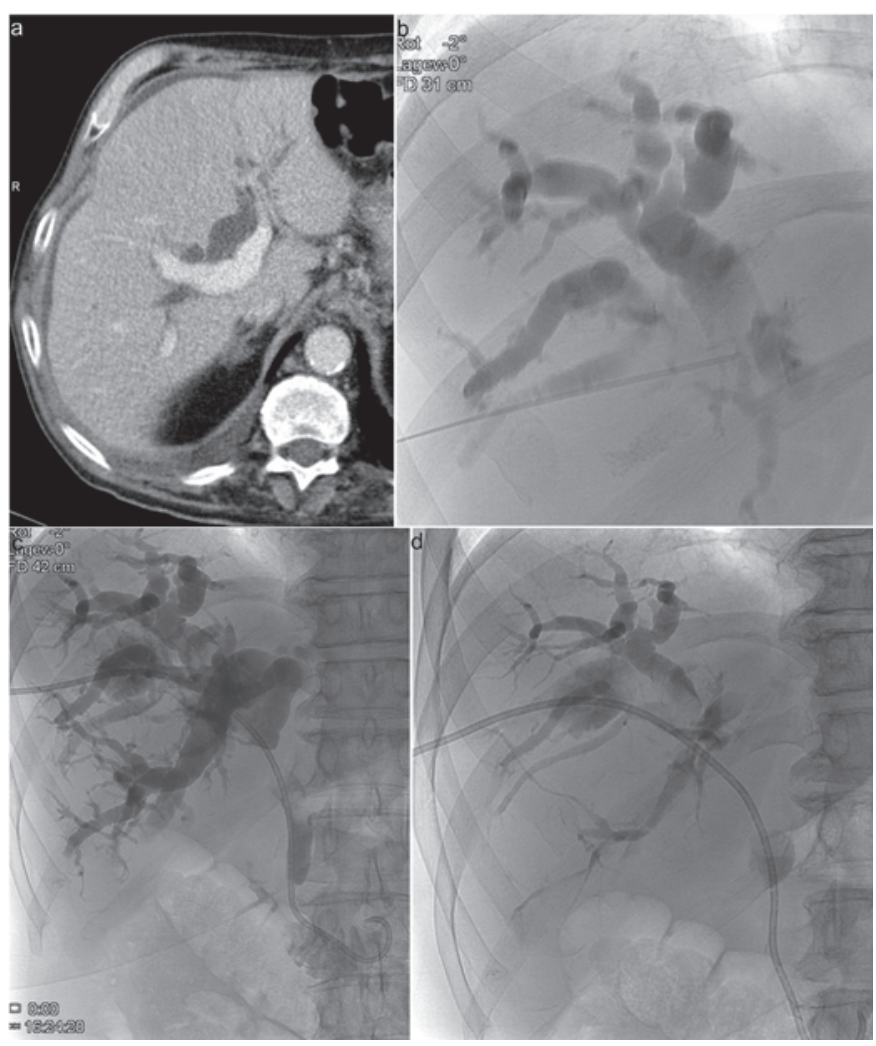

Fig. 1. a Initial situation: dilated biliary ducts in computed tomography. b Fluoroscopy of puncture of dilated duct guided with sonography and cholangiography. c Insertion of percutaneous transhepatic biliary drainage. d Pigtail drain ends enterically. Visible decontrasting of the biliary ducts.

Percutaneous biliary interventions have become less frequent due to the remarkable progress in endoscopic techniques. In most cases, endoscopic procedures are easily performed without major or minor complications as well as a lack of potential risks of percutaneous puncture [9]. Evaluation of the biliary anatomy using percutaneous transhepatic cholangiography (PTC) is indicated if endoscopic retrograde cholangiopancreaticography (ERCP) fails in the presence of biliary leakage and obstruction, if ERCP is unlikely to succeed, or if the anatomical conditions after surgery such as hepaticojejunostomy make direct access impossible [11, 13-15]. PTBD is the only endoluminal treatment option of biliary leakage if the duodenum or the common bile duct cannot be cannulated [13]. It gives the opportunity to decompress the biliary system, redirect the bile flow from the defect into the bile ducts while secondarily allowing the leak to heal, and to avoid further surgery or as a bridging therapy while stabilizing the patient's condition prior to surgery [13]. The principle of healing a bile leak is to create a low-pressure system along the biliary tract [13]. PTC is performed prior to PTBD. Prior to PTC, imaging of the biliary system using magnetic resonance cholangiopancreatography, ultrasound, or CT, depending on availability, is extremely helpful in planning the procedure (fig. 1a).

Cross-sectional imaging can provide further information about the pattern of biliary duct dilatation as well as anatomy and level of leakage $[6,13]$. Allergic reactions to contrast media and large volumes of ascites are relative contraindications [13] due to insuf- 
Fig. 2. Approach to treat biliary duct leak. After primary percutaneous transhepatic biliary drainage (a), insertion of a guide wire through the drainage (b) and removal of drainage. Implantation of stent over the leak (c) and controlling of the closure of the leak.
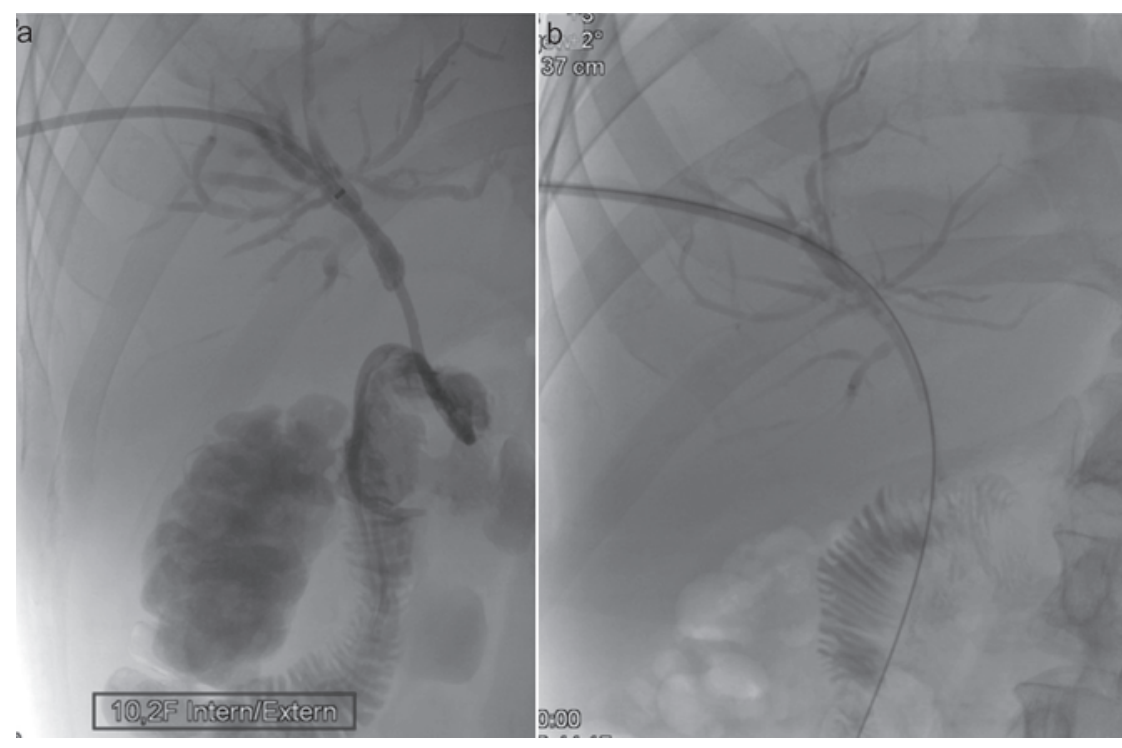

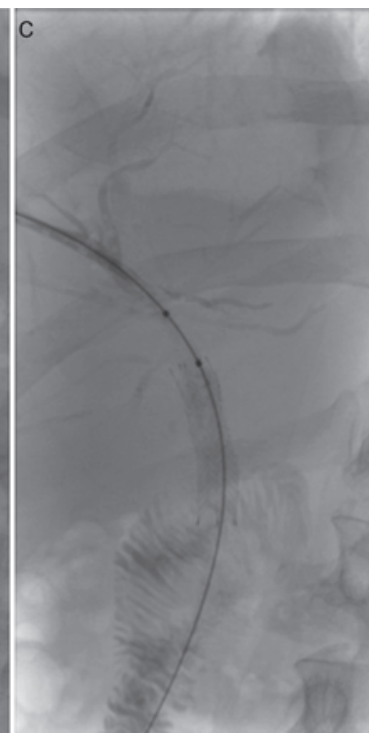

ficient fixation of the liver to the abdominal wall and a higher risk of procedure-related bleedings [14]. The C-arm should rotate freely around the level of the liver [15]. The targeted duct should be adjacent to the portal vein and artery [16]. The puncture site depends on the decision to either puncture the right duct (access would be in the mid-axillary line in the intercostal space caudal to the costophrenic sulcus) or the left duct (epigastric approach without risk of crossing the pleura). The targeted biliary duct is best located by ultrasound $[13,14,16]$. A 21 - or 22-gauge Chiba needle is then passed through the liver while visualizing the entire passage in plane of the transducer to the duct (fig. 1b) $[13,16]$. Overinjection of contrast media should be avoided due to sepsis during PTC [14]. Depending on the images of the cholangiography, either multiple images in varied projections are made or therapy follows [14].

The first usage of PTBD was as early as 1974 [12], and before the development of endoscopic procedures it was a frequently used interventional treatment for biliary obstruction [12, 14]. Based on the cholangiography images of PTC, a corresponding biliary duct to reach the leak is chosen; then, it is either punctured by using a broad puncture or the already placed puncture needle is used. After removal of the puncture needle, insertion of a guide wire follows which is then pushed through the anastomosis distal to the leak into the bowel. Now exchange of the obturator to a $4 \mathrm{~F}-5 \mathrm{~F}$ catheter and placement along the guide wire into the bowel is carried out, usually in the jejunum after hepaticojejunostomy or otherwise duodenal $[12,14,16]$. Exchange of the flexible guide wire for a stiffer wire is followed by switching the $4 \mathrm{~F}-5 \mathrm{~F}$ catheter to a drainage (up to $12 \mathrm{~F}$ ) with 16 or 32 holes (fig. 1c) [13, 14]. Catheter holes must be located on both sides of the bile leak in order to keep pressure as low as possible $[6,13]$. After angiographic control of location (fig. 1d), the drain is fixed to the skin. Percutaneous biliary drainages are irrigated after intervention; however, the irrigation needs to be performed gently in order to ensure that there is no increase of pressure, which might keep the leak open [16].
Periodic cholangiograms show changes in biliary extravasation, and if no further biliary leak is visible, the drain is changed to a catheter without any holes and with a smaller lumen [13]. This catheter is left in place for $4-8$ weeks to enable access to the biliary system [13]. Later on, the catheter may be removed if no bile duct obstruction or re-leakage occurs [13]. Despite treating the leakage by external biliary drainage, implantation of covered stents is an option to seal the leak and to provide biliary drainage [13, 17]. The covered stent is inserted either at primary PTC or during a secondtime approach after PTBD (fig. 2a, b) [13]. In order to place the stent safely, the length of the leakage, landing zones, and diameter of the biliary duct need to be defined. Stents of 7-10 mm diameter are usually appropriate for intrahepatic ducts (fig. 2c) [13]. Selfexpandable covered stents have to be used to avoid balloon dilatation with the risk of increasing the leakage. Just like in endoscopic stent implantation, self-expandable plastic stents are usually sufficient for the treatment of biliary leaks [17]. Fully covered metal stents might be an option for complex leaks. There is not enough data comparing fully covered metal stents to covered plastic stents in the percutaneous treatment of bile leakage. Stent migration is one complication after PTBD. There is also not enough data available regarding the possibility of re-leakage after stent migration.

In patients with non-dilated ducts, technical success of PTC has been reported between 65 and $90 \%[6,13,16,18]$. Success rates of PTBD for biliary leakage are reported to be $50-100 \%$ without secondary surgery [13]. Success rates should be higher than 95\% in patients with dilated ducts [16]. The average time range from PTBD to closure of the leak is described as 9-150 days [13]. In patients with biliary leakage, the biliary ducts are often non-dilated compared to biliary obstruction and the complication rate is reported to be higher [13].

The rate of major complications after PTC and PTBD is 2\% [11] to $4 \%$ [6] and up to $25 \%$ [13], but there is a lack of differentiation between major and minor complications according to Portugaller and Hausegger [13]. According to Hunter [16], a major complica- 
tion rate of $4 \%$ or higher should induce a review of PTC technique. Hemorrhage secondary to arterial injury as well as subcapsular liver hematoma, residual biliary stenosis, ductal perforation, and metabolic acidosis from continuous bile loss have been reported $[13,16]$. A minor complication could be fever with chills [13]. There is controversy about when to remove the biliary drainage catheter [13]. Some authors describe removing the catheter when cholangiography confirms healing of the leak without stenosis, whereas other authors keep the catheter in situ for a longer time [13]. So far, no advice can be given when to remove the catheter [13]. Higher drainage catheter lumen (12F) might prevent secondary biliary stenosis after leak [13].

\section{Conclusion}

PAD, PTBD, and PTC are long-known, safe, and well-established procedures for the treatment of complications after hepaticojejunostomy, other abdominal surgery, and biliary duct injuries. While $\mathrm{PAD}$ is a standard procedure in interventional radiology, PTC and PTBD are more difficult and only applied if ERCP is not possible. Covering biliary leakage by percutaneous self-expanding stents is a safe procedure with the risk of stent migration. PTBD is the more common percutaneous treatment of biliary leakage by means of creating low pressure along the biliary system. However, it requires a healing time of up to several weeks.

The interventions can be performed either as a therapeutic option or as a bridging procedure before surgery. Success rates of up to $93 \%$ for PAD [8] and of $60-90 \%$ for PTC and PTBD $[6,16]$ are reported. Mortality rates vary between 0.7 and $6 \%$ (PAD) $[8,6]$. Major complications are described in less than 2.5\% (PBD) [16]. Typical indications of PAD, PTC, and PTBD after hepaticojejunostomy are epigastric abscesses and leakage. Uncontrollable coagulation status and lack of a safe access route are the only severe contraindications. Patient selection and an interdisciplinary approach are the keys to successful intervention.

\section{Disclosure Statement}

Katharina May and Peter Hunold have nothing to disclose.

\section{References}

1 Mir F: Hepaticojejunostomy. http://emedicine.medscape com/article/1892165-overview (last access: 07.03. 2017).

2 Sarmiento J: Hepaticojejunostomy: indications and surgical technique; in van Heerden JA, Farley DR (eds): Operative Techniques in General Surgery. Biliary/Duodenal Bypass Procedures. Amsterdam, Elsevier, 2000, pp 295-303.

3 Solomkin JS, Mazuski JE, Bradley JS, Rodvold KA Goldstein EJC, Baron EJ, O’Neill P, Chow AW, Dellinger EP, Eachernpati SR, Gorbach S, Hilfiker M, May AK, Nathens AB, Sawyer RG, Bartlett JG: Diagnoses and Management of Complicated Intra-Abdominal Infection in Adults and Children: Guidelines by the Surgical Infection Society and Infectious Diseases Society of America. CID 2010;50:133-164. www.uphs. upenn.edu/bugdrug/antibiotic_manual/sisidsaintraabdominalinfection2010.pdf.

4 Cinat ME, Wilson SE, Din AM: Determinants for successful percutaneous image-guided drainage of intraabdominal abscess. Arch Surg 2002;137:845-849.

5 Kos S, Jakob AL: Perkutane Abszessdrainagen. Radiologie up2date 2008;2:107-131.

6 Thabet A, Arellano RS: Catheter drainage of abdominal abscesses and fluid collections; in Kandarpa K, Machan L (eds): Handbook of Interventional Radiologic Procedures, ed 4. Philadelphia, Lippincott Williams \& Wilkins, 2011, pp 527-537.
7 Lorenz J, Thomas JL: Complications of percutaneous fluid drainage. Semin Intervent Radiol 2006;23:194204.

8 Wiggermann A, Hunold P: Radiologisch-interventionelle Drainage bei abdomineller Sepsis. Viszeralmedizin 2013;29:14-20.

9 Saber AA, LaRaja RD: Abdominal Abscess Treatment \& Management. http://emedicine.medscape.com/article/ 1979032-treatment\#d11 (last access: 02.03.2017).

10 Johnson WC, Gerzof SG, Robbins AH, Nabseth BC: Treatment of abdominal abscesses: comparative evaluation of operative drainage versus percutaneous catheter drainage guided by computed tomography or ultrasound. Ann Surg 1981;194:510-519.

11 Thompson CM, Saad NE, Quazi RR, Darcy MD, Picus DD, Menias CO: Management of iatrogenic bile duct injuries: role of the interventional radiologist. Radiographics 2013;33:117-134.

12 Dawood A: Percutaneous biliary drainage. http:// emedicine.medscape.com/article/1828052-overview (last access: 07.03.2017).

13 Portugaller RH, Hausegger KA: Management of biliary leaks; in Mauro MA, Murphy KPJ, Thomson KR, Venbrux AC, Morgan RA (eds): Image-Guided Interventions, ed 2. Philadelphia, Saunders Elsevier, 2014, pp 1002-1007.
14 Rieber A, Brambs HJ: Perkutane transhepatische Drainage; in Görich J, Brambs H-J: Interventionelle minimal-invasive Radiologie. Referenz-Reihe Radiologie. Stuttgart, Thieme, 2001, pp 58-73.

15 Dawood A: Percutaneous transhepatic cholangiography. http://emedicine.medscape.com/article/1828033overview (last access: 07.03.2017).

16 Hunter DW: Percutaneous biliary interventions; in Kandarpa K, Machan L (eds): Handbook of Interventional Radiologic Procedures, ed 4. Philadelphia, Lippincott Williams \& Wilkins, 2011, pp 577-590.

17 Pausawasadi N, Soontornmanokul T, Rerknimitr R: Role of fully covered self-expandable metal stent for treatment of benign biliary strictures and bile leaks. Korean J Radiol 2012;13(suppl 1):S67-S73.

18 Hatzidakis AA: Quality Improvement Guidelines for Percutaneous Transhepatic Cholangiography and Biliary Drainage. Vienna, Cardiovascular and Interventional Radiological Society of Europe, 2005. https://eucsite-storage-prod.s3.amazonaws.com/www-cirse-org/ files/files/SOP/2005/SOP_CIRSE_2005_Percutaneous\%20Transhepatic\%20Cholangiography\%20and\%20 Biliary\%20Drainage.pdf. 